\title{
SITUAÇÕES DE VULNERABILIDADES EM SAÚDE VIVENCIADAS POR TRABALHADORAS SEXUAIS EM TEMPOS DE PANDEMIA DA COVID-19
}

\author{
SITUATIONS OF HEALTH VULNERABILITIES \\ EXPERIENCED BY SEX WORKERS IN TIMES OF \\ COVID-19 PANDEMIC
}

\section{SITUACIONES DE VULNERABILIDADES EN SALUD EXPERIMENTADAS POR LAS TRABAJADORAS SEXUALES EN TIEMPOS DE PANDEMIA DE COVID-19}

\author{
Pablo Luiz Santos Couto ${ }^{1}$ \\ Antônio Marcos Tosoli Gomes ${ }^{2}$ \\ Samantha Souza da Costa Pereira ${ }^{3}$ \\ Alba Benemérita Alves Vilela ${ }^{4}$ \\ Tarcísio da Silva Flores ${ }^{5}$ \\ Carle Porcino ${ }^{6}$
}

Como citar este artigo: Couto PLS, Gomes AMT, Pereira SSC, Vilela ABA, Flores TS, Porcino C. Situações de vulnerabilidade em saúde vivenciadas por trabalhadoras sexuais em tempos de pandemia da COVID-19. Rev baiana enferm. 2021;35:e37327.

Objetivo: refletir sobre as situações de vulnerabilidades em saúde vivenciadas por trabalhadoras sexuais durante a pandemia decorrente da infecção pelo SARS-CoV-2. Método: trata-se de estudo reflexivo, fundamentado no referencial teórico conceitual da vulnerabilidade em quatro dimensões: ontológica, epidemiológica, simbólica e político-programática. Resultado: a saúde das profissionais do sexo, na experiência cotidiana do trabalho sexual, tem sido um desafio para os governos entre todos os grupos associados a estigmas e populações vulneráveis, justamente por serem marginalizados socialmente. Isso se configura como importante para a compreensão desse fenômeno em meio à crise financeira, econômica e de saúde que pessoas de diferentes nações sofrem diante do progresso da SARS-CoV-2. Conclusão: as situações de vulnerabilidade em saúde decorrente da infecção pelo SARS-CoV-2 que expõem as profissionais do sexo são consolidadas, principalmente, pela ausência do Estado na proteção sócio legal e na garantia dos direitos humanos, para que essas mulheres adquiram condições de enfrentamento e prevenção.

Descritores: Trabalhadoras Sexuais. COVID-19. Coronavírus. Vulnerabilidade em Saúde. Populações Vulneráveis.

\footnotetext{
Enfermeiro. Coordenador e Docente do Colegiado de Enfermagem do Centro Universitário FG. Guanambi, Bahia, Brasil. pabloluizsc@hotmail.com. http://orcid. org/0000-0002-2692-9243.

Enfermeiro. Doutor em Enfermagem. Docente do Programa de Pós-Graduação em Enfermagem da Universidade do Estado do Rio de Janeiro. Rio de Janeiro, Rio de Janeiro, Brasil. http://orcid.org/0000-0003-4235-9647.

3 Enfermeira. Mestra em Saúde Coletiva. Docente do Colegiado de Enfermagem do Centro Universitário FG. Guanambi, Bahia, Brasil. http://orcid.org/0000-000 I5978-520X.

4 Enfermeira. Doutora em Enfermagem. Docente do Programa de Pós-Graduação em Enfermagem e Saúde da Universidade Estadual do Sudoeste da Bahia. Jequié, Bahia, Brasil. http://orcid.org/0000-0002-I I87-0437.

5 Advogado. Especialista em Direito Trabalhista e Previdenciário. Colegiado de Direito do Centro Universitário FG. Guanambi, Bahia, Brasil. http://orcid.org/0000000 I-6350-2698.

6 Psicóloga. Mestra em Estudos Interdisciplinares da Universidade Federal da Bahia. Salvador, Bahia, Brasil. http://orcid.org/0000-000 I-6392-029I.
} 
Objective: to reflect on the situations of health vulnerabilities experienced by sex workers during the pandemic resulting from SARS-CoV-2 infection. Method: this is a reflective study, based on the conceptual theoretical framework of vulnerability in four dimensions: ontological, epidemiological, symbolic and political-programmatic. Result: the bealth of sex workers, in the daily experience of sexual work, has been a challenge for governments among all groups associated with stigmas and vulnerable populations, precisely because they are socially marginalized. This is important for understanding this phenomenon in the midst of the financial, economic and health crisis that people from different nations suffer before the progress of SARS-COV-2. Conclusion: the situations of health vulnerability resulting from the SARS-COV-2 infection that expose sex workers are consolidated, mainly, by the absence of the State in the social-legal protection and in the guarantee of human rights, so that these women acquire conditions of coping and prevention.

Descriptors: Sex Workers. COVID-19. Coronavirus. Health Vulnerability. Vulnerable Populations.

Objetivo: reflexionar sobre las situaciones de vulnerabilidades en salud experimentadas por las trabajadoras sexuales durante la pandemia resultante de la infección por SARS-CoV-2. Método: se trata de un estudio reflexivo, basado en el marco teórico conceptual de vulnerabilidad en cuatro dimensiones: ontológica, epidemiológica, simbólica y politica-programática. Resultado: la salud de las trabajadoras sexuales, en la experiencia diaria del trabajo sexual, ha sido un desafio para los gobiernos entre todos los grupos asociados con estigmas y poblaciones vulnerables, precisamente porque están socialmente marginadas. Esto es importante para entender este fenómeno en medio de la crisis financiera, económica y de salud que sufren personas de diferentes naciones ante el progreso del SARS-CoV-2. Conclusión: las situaciones de vulnerabilidad a la salud resultantes de la infección por SARS-COV-2 a que exponen las trabajadoras sexuales se consolidan, principalmente, por la ausencia del Estado en la protección social-jurídica $y$ en la garantía de los derechos humanos, para que estas mujeres adquieran condiciones de enfrentamiento $y$ prevención.

Descriptores: Trabajadoras sexuales. COVID-19. Coronavirus. Vulnerabilidad en Salud. Poblaciones Vulnerables.

\section{Introdução}

A corona virus disease (COVID-19) é decorrente da infecção pelo coronavírus SARS-CoV-2 que ganhou caráter de pandemia após seu surto ter sido detectado incialmente na província chinesa de Hubei ${ }^{(1)}$, tendo se alastrado rapidamente por todos os países, incluindo o Brasil. As medidas de prevenção implementadas pelos governos e a detecção precoce têm se consistido em desafio, visto que o vírus se alastra de maneira rápida e muitas vezes de modo assintomático, ao mesmo tempo em que a falta de exames diagnósticos e a incapacidade dos serviços de saúde em absorver a população com complicações da virose, potencializa o aumento da mortalidade, sobretudo em grupo de pessoas que vivenciam o abandono do Estado ${ }^{(1-2)}$.

Apesar dos órgãos internacionais como a Organização Mundial de Saúde (OMS) declararem que a tendência de complicações da doença (Síndrome da Angústia Respiratória Grave) é perceptível em pessoas idosas e naquelas que sofrem/convivem com doenças crônicas não transmissíveis ${ }^{(3)}$, os grupos de populações marginalizadas pela sociedade são ainda mais vulneráveis.

A noção de vulnerabilidade surgiu ainda no início da década de 1990, por epidemiologistas de Harvard, no intuito de romper com o significado culpabilizador que o termo "risco" remetia às pessoas gays, profissionais do sexo e usuários de drogas injetáveis em processo de adoecimento decorrente da epidemia do Vírus da Imunodeficiência Humana/Síndrome de Imunodeficiência Adquirida (HIV/aids), que passou a acometer outros grupos sociais que não se enquadravam no chamado grupo que tinham comportamentos de risco: crianças, adolescentes, mulheres e idosos, por exemplo ${ }^{(4-5)}$.

No Brasil, o marco teórico da vulnerabilidade passou a ser discutido em apenas três dimensões: a individual, a social e a programática. Contudo, diante da necessidade de ampliação do conceito para explicar as condições que tornam as pessoas vulneráveis e com dificuldades de 
enfrentamento de tais situações, há necessidade de entender o conceito com base em outras perspectivas menos estanques e culpabilizatórias, no que concerne aos demais agravos do processo saúde-doença ${ }^{(5)}$.

Desse modo, para este estudo, o conceito de vulnerabilidade pode ser entendido como a condição na qual as pessoas ou grupos sociais (neste caso, as profissionais do sexo) vivenciam seus cotidianos e se expõem a situações que interferem no processo saúde-doença, em diversos níveis, em decorrência das falhas na atenção do Estado e da sociedade, interferindo no enfrentamento de determinadas situações da vida ${ }^{(4)}$. Essas mulheres exercem seu trabalho com alto grau de vulnerabilidade e ainda marginalizado pela sociedade, por estar relacionado à esfera da sexualidade e das práticas sexuais como modo de obtenção de lucro, sendo envolta em estigmas sociais $^{(6-8)}$. Essa maior vulnerabilidade se dá por não possuir amparo do Estado, pela necessidade de se expor para manter a sobrevivência, e pelas condições de moradia facilitar o contágio.

O governo da Argentina é um exemplo de apoio às trabalhadoras sexuais no enfrentamento à pandemia provocada pela infecção do SARS-CoV-2, pois conforme a Associação de prostitutas do país, o Estado tem se mostrado presente ao conceder um auxílio mensal de 5.000 pesos, o que equivale a 500 reais. Ainda assim, o estigma é algo que elas temem, visto que, ao acessar o benefício social, constará no banco de dados, que se tornará público e, por esse motivo, muitas famílias passariam a ter ciência da sua profissão ${ }^{(9)}$.

As situações de vulnerabilidades enfrentadas pelas trabalhadoras sexuais em tempos de pandemia da COVID-19 extrapolam às Infecções Sexualmente Transmissíveis (IST) e se acentuam à medida que elas se sentem negligenciadas e abandonadas pelo Estado, sem a proteção trabalhista, como a garantia de renda mínima para seu sustento, uma vez que com a implementação de medidas de isolamento social e quarentena, encontram-se impossibilitadas de realizarem seus serviços, em decorrência da diminuição ou ausência de clientes ${ }^{(10)}$.
Dessa forma, objetivou-se refletir sobre as situações de vulnerabilidades em saúde vivenciadas por trabalhadoras sexuais nesse período de pandemia decorrente da infecção pelo SARS-CoV-2.

\section{Método}

A análise reflexiva foi desenvolvida mediante discussão transversalizada e articulada entre quatro dimensões da vulnerabilidade (ontológica, epidemiológica, simbólica, político-programática) e a forma como o Estado e a sociedade têm se posicionado e preparado para implementar medidas protetivas e preventivas, que visam minimizar as situações de vulnerabilidades em saúde, que tornam as trabalhadoras sexuais mais suscetíveis ao adoecimento decorrente pela COVID-19. Esse método possibilita contribuir para a fundamentação sobre um tema, fenômeno ou objeto que necessita de maiores aprofundamentos, como é o caso do curso da COVID-19 a grupos populacionais vulneráveis e estigmatizados, como as trabalhadoras sexuais.

\section{Resultados e discussão}

A reflexão permitiu compreender acerca das situações de vulnerabilidades em saúde das profissionais do sexo, no contexto de pandemia pelo vírus Sars-Cov-2, bem como os impactos para esse público, no que concerne à invisibilidade perpetrada pelo Estado e sociedade. Esse cenário impacta na prevenção e na adoção de medidas protetivas, referente ao avanço da COVID-19 às pessoas desamparadas e invisibilizadas por governos, para implementação de políticas de inclusão, segurança e garantia dos direitos humanos e trabalhistas básicos.

\section{A vulnerabilidade ontológica das profissionais do sexo: do individual ao coletivo e a importância da educação em saúde}

A vulnerabilidade ontológica refere-se à vulnerabilidade característica de todo o ser humano pelo fato de existir e estar vivo ${ }^{(11)}$. Nesse sentido, 
é o reconhecimento da fragilidade humana presente de forma indiscriminada em todos os seres humanos, em qualquer contexto, sociedade e nível cultural e educacional, por exemplo. Apresenta-se como um fato inerente à sua evolução bio-psico-socio-espiritual que, em paralelo à sua grande complexidade, também apresenta importante fragilidade diante de situações, eventos e relações. De maneira mais prática, caracteriza-se pela fragilidade humana de adoecer, sofrer e morrer, consubstanciada pela incerteza permanente que se encerra no futuro mediato ou imediato ${ }^{(12-13)}$.

Em contraposição à constatação de sua vulnerabilidade ontológica, o que igualaria todos os seres que estão sob as dimensões do tempo e do espaço, os seres humanos possuem a consciência do seu ser vulnerável e do seu estado de vulnerabilidade ${ }^{(13)}$. Esse equilíbrio complexo entre a vulnerabilidade como condição do ser que se é, e a consciência dela, permite maiores estruturas, ao menos em princípio, para o seu enfrentamento e a redução dos seus níveis ${ }^{(14-15)}$.

Dessa maneira, há uma vulnerabilidade à COVID-19 por parte das profissionais do sexo, que se relaciona ao fato de serem seres humanos, inscritas no quadro da degeneração orgânica que caracteriza todos os seres sobre a Terra e se desdobra não somente em sua natureza somática e orgânica, mas também a psicológica e a espiritual, por exemplo, além de outras que poderiam ser citadas. A imagem mais clara da vulnerabilidade ontológica refere-se à uma criança nos primeiros anos da sua vida que, se for abandonada, não terá condições de sobreviver, em face aos desafios que enfrentará, o que a difere de animais, que precocemente apresentam ferramentas corporais e instintivas que os capacitam o mais cedo possível ${ }^{(11,13)}$.

\section{Vulnerabilidade e Epidemiologia: para a compreensão do trabalbo sexual desempenhado por mulheres}

Nos últimos tempos, no processo de se compreender e entender a saúde da sociedade, têm-se buscado trazer a discussão de alguns conceitos que demonstram contribuir na construção do saber e da importância da saúde comunitária. Nesse contexto, aponta-se aqui uma discussão breve do que vem a ser vulnerabilidade conjuntamente com a epidemiologia, o que pode trazer mais elementos na compreensão do trabalho das trabalhadoras sexuais.

A dimensão epidemiológica da vulnerabilidade das trabalhadoras sexuais pode estar ligada aos indicadores em saúde, que apontam para o aumento ou não do agravo a esse grupo populacional e remete às consequências do comportamento que elas adotam para se protegerem da infecção pelo coronavírus, muitas vezes estereotipadas, por ser uma dimensão confundida com a individual, visto que leva à ideia de culpabilização e manutenção dos estigmas ${ }^{(8,10,16)}$.

Destarte, a vulnerabilidade vai remeter à fragilidade, termo presente em diferentes campos temáticos, mas muito especificamente no dos Direitos Humanos ${ }^{(17)}$. Corrobora aqui, no que tange aos enfrentamentos das profissionais do sexo que, no Brasil, a vulnerabilidade remete à deterioração dos direitos civis, perda de garantias adquiridas e fragilização da cidadania, potencializada pelas questões de gênero e classe ${ }^{(8,18)}$. São ainda destacados: a desigualdade perante a lei e sujeição à violência, dificuldade de acesso à moradia, aos serviços de saúde, à assistência social e ao emprego, a coexistência de modalidades arcaicas e modernas de trabalho com importante participação da informalidade, isto é, não são legalmente protegidas e equivale ao apartheid social nos ambientes urbanos nos quais essas profissionais do sexo estão inseridas ${ }^{(19)}$.

Nesse sentido, algumas reflexões acerca de vulnerabilidade, enquanto referência para a consideração desse conceito na Epidemiologia e Saúde Coletiva brasileiras, propõe-se que ele seja vinculado à garantia da cidadania de populações politicamente fragilizadas na perspectiva dos direitos humanos ${ }^{(15,20-21)}$.

\section{Vulnerabilidade simbólica: das construções sociais de gênero aos estigmas sociais}

O nível simbólico relaciona-se às iniquidades sociodemográficas, de escolaridade, religiosas, culturais, comunitárias, às questões de 
gênero e aquelas relacionadas à interferência na vontade dos grupos de profissionais e das pessoas na possibilidade de realizar ações de promoção, prevenção e proteção da infecção ao coronavírus, uma vez que tais iniquidades influenciam ou determinam construções sociais e históricas dos papéis desempenhados por homens e mulheres ${ }^{(18)}$. Destaca-se que os espaços a serem ocupados por cada um numa sociedade machista e patriarcal, como a brasileira, nos quais as trabalhadoras sexuais extrapolam por utilizar o sexo como fonte de renda e ocupar os espaços públicos, constituem-se como duas situações simbólicas e práticas "proibidas" para as mulheres ${ }^{(6-8,24)}$.

As questões simbólicas que envolvem o trabalho sexual as tornam vulneráveis, pois muitas sofrem o preconceito institucional de algumas pessoas de setores da saúde, já que carregam o estigma de utilizarem a prática sexual como ocupação. Além disso, é contestada pelas trabalhadoras a forma como a sociedade as insere nos grupos vulneráveis, pois se protegem, se cuidam e se previnem de IST $^{(6,25)}$. Deve-se destacar ainda, que boa parte das trabalhadoras sexuais não consegue desenvolver atividades de Home Office, em função da presença de familiares, e inclusive em alguns casos, estes nem terem ciência do trabalho desenvolvido por elas ${ }^{(8,10,26)}$.

Mesmo com a liberdade que tomam para $\mathrm{si}$, as profissionais do sexo estabelecem limites simbólicos entre a vida pessoal e profissional, determinando o que pode ou não pode durante as relações sexuais. A relação profissional desenvolvida com os clientes tem o intuito de fazer com que eles alcancem o prazer e elas ganhem o valor por elas estabelecido, o que revela o modo como elas levam a vida profissional e desenvolvem "contrato sexual" firmado com os homens ${ }^{(27-28)}$.

Muitas mulheres sofrem preconceito por optarem vivenciar a prática sexual de modo livre e como desejam, por entenderem que seus corpos não possuem donos (elas são donas de si), e o fato de serem trabalhadoras sexuais, o preconceito é potencializado, diferente das mulheres que seguem os padrões tradicionais quando são ensinadas a casar e a terem sua sexualidade restrita ao privado dentro do matrimônio ${ }^{(18,25)}$. Evidenciou-se, em estudo desenvolvido na França, com prostitutas, o fato da sociedade considerar aceitável a troca do orgasmo pelas declarações que os homens fazem às suas companheiras e julgarem as outras que escolhem valorar o sexo e cobrar pela satisfação alcançada por ele ${ }^{(29)}$.

Como ocorre historicamente às minorias silenciadas e socialmente invisíveis, uma situação de crise, seja ela de qualquer natureza (econômica, sanitária, política, dentre outras) afetará mais intensamente esses grupos, na esfera das sociedades patriarcais e machistas. Corroborando essa ideia, Simone de Beauvoir considera que "Nunca se esqueça de que basta uma crise política, econômica ou religiosa para que os direitos das mulheres sejam questionados $[\ldots]^{124: 8)}$. $\mathrm{O}$ direito às condições dignas de trabalho e ao usufruto de recursos financeiros que possibilitem a satisfação de suas necessidades mais vitais, são, tradicionalmente, negados às trabalhadoras sexuais, e isso se agudiza sobremaneira no contexto da quarentena pela COVID-19.

\section{Vulnerabilidade politico/programática das profissionais do sexo: o desproporcional impacto da crise sobre as camadas excluidas}

A dimensão política/programática do conceito de vulnerabilidade pode ser compreendida mediante a verificação da existência de políticas públicas, programas, ações e iniciativas, que perfazem os determinantes da conjuntura (social, de saúde, ou outros) que se pretende modificar. Diz respeito também ao comprometimento institucional com a prevenção de agravos à saúde dos sujeitos individuais e coletivos ${ }^{(5,30)}$.

Nos últimos 20 anos, as consequências de epidemias e pandemias mais recentes (como Zika vírus, Ebola, pandemia de H1N1 e síndrome respiratória do Oriente Médio, por exemplo) revelam a necessidade de fortalecer os setores de saúde pública nacional com recursos pessoais qualificados, capacidade de absorção de demanda em crescimento exponencial e 
infraestruturas, incluindo sistemas de vigilância de doenças e redes de laboratórios para atender às populações negligenciadas e estigmatizadas, como as prostitutas ${ }^{(2-3,10,16)}$.

Como cidadãs, à semelhança dos demais trabalhadores, aposentados, desempregados e pessoas que não trabalham, as prostitutas carregam sobre si uma carga tributária e, portanto, deveriam usufruir de alguns dos direitos que lhe são negados $^{(23)}$, como jornada de trabalho definida por lei, benefícios previdenciários advindos da sua contribuição laboral, bem como usufruto de piso salarial, terço de férias e o seu gozo, décimo terceiro salário, seguro desemprego e o auxílio doença. Não há sequer uma tentativa de diálogo entre representantes do governo com a Central Única de Trabalhadoras Sexuais (CUTS) no Brasil, para repensar as estratégias de enfrentamento às vulnerabilidades potencializadas pelo SARS-COV-2 ${ }^{(30)}$.

Outrossim, em contrapartida, o Programa Conjunto das Nações Unidas sobre HIV/Aids (UNAIDS) e a Rede Global de Projetos de Trabalho Sexual, no intuito de garantir o cumprimento dos direitos humanos, orientam a implementação de medidas protetivas e o respeito à classe, em especial neste período de dificuldades geradas pela pandemia da COVID-19 e enfrentada por toda a sociedade que vive do trabalho informal, aí incluídas as trabalhadoras sexuais. Estas ainda têm que conviver com o aumento do assédio por parte dos clientes, a redução da renda e a exclusão de planos emergenciais de proteção social $^{(25,30)}$.

Baseando-se nos princípios dos direitos humanos, também é proposto pelas entidades e órgãos supracitados que os governos criem um firewall de emergência, articulado entre os serviços de saúde e as autoridades de imigração, garantido às profissionais do sexo, que fazem migração, ter acesso aos serviços de saúde. Garante ainda o acesso financeiro destinado pelos programas de apoio emergencial, acesso a moradias destinadas aos sem-teto, incluindo as trabalhadoras que não possuem lar, interrupção de prisões e processos das mulheres que oferecem o serviço sexual nos países cujo trabalho sexual é criminalizado, envolver as comunidades e os órgãos de classe das prostitutas no planejamento da saúde pública e ofertas testes para a COVID-19, livre do preconceito institucional ${ }^{(30)}$.

\section{Conclusão}

Conclui-se que as quatro dimensões da vulnerabilidade aqui analisadas, a ontológica, a epidemiológica, a simbólica e a político-programática, revelam que as situações de vulnerabilidade à COVID-19, às quais as trabalhadoras sexuais estão expostas, perpassam sobretudo pela ausência do Estado na proteção sócio legal e na garantia dos princípios dos direitos humanos. Reconhece-se ainda, que a vulnerabilidade se relaciona à manutenção de estigmas e de preconceitos sociais e institucionais, reforçando a marginalização dessas mulheres que compõem esse grupo social, bem como a invisibilidade para políticas públicas necessárias às suas necessidades, seja nesse período de pandemia, seja em qualquer época.

Tais questões dificultam a adoção, por parte delas, de habilidades/condutas de enfrentamento à pandemia, uma vez que as iniquidades sociais, culturais e de gênero terminam por adquirir maior magnitude em sociedades governadas por pessoas inscritas no âmbito do patriarcado, da moralidade e da tradição cristã, o que gera, como consequência, a invisibilidade da mulher, sobretudo daquelas que vivenciam a liberdade sexual e utilizam a prática sexual como um serviço para a obtenção de renda. Nesse momento em que o isolamento e o distanciamento social são práticas preventivas importantes de combate à infecção pelo SARS-CoV-2, as profissionais do sexo têm redução na procura de clientes e, consequentemente, no lucro e no suprimento das suas necessidades e de seus familiares.

Nesse contexto de enfrentamento das situações de vulnerabilidade pelas trabalhadoras sexuais, nas diversas dimensões analisadas, deve-se destacar que o sistema político e social são os principais responsáveis por dificultar a proteção de cada uma delas, visto que há iniquidades de gênero, econômicas e ocupacionais 
envolvidas, além da falta de organização e apoio do Estado na proteção e segurança às trabalhadoras sexuais que propiciem condições dignas de proceder com isolamento, alimentação adequada e condições de higiene diante do avanço do vírus SARS-CoV-2.

\section{Colaborações:}

1 - concepção, projeto, análise e interpretação dos dados: Pablo Luiz Santos Couto, Antonio Marcos Tosoli Gomes, Samantha Souza da Costa Pereira, Alba Benemérita Alves Vilela, Tarcísio da Silva Flores e Carle Porcino;

2 - redação do artigo e revisão crítica relevante do conteúdo intelectual: Pablo Luiz Santos Couto, Antonio Marcos Tosoli Gomes, Samantha Souza da Costa Pereira, Alba Benemérita Alves Vilela, Tarcísio da Silva Flores e Carle Porcino;

3 - aprovação final da versão a ser publicada: Pablo Luiz Santos Couto, Antonio Marcos Tosoli Gomes, Samantha Souza da Costa Pereira, Alba Benemérita Alves Vilela, Tarcísio da Silva Flores e Carle Porcino.

\section{Referências}

1. World Health Organization. Emergency Committee. Statement on the second meeting of the International Health Regulations (2005) Emergency Committee regarding the outbreak of novel coronavirus (COVID-19) [Internet]. Geneva (CHE); 2020 [cited 2020 Mar 27]. Available from: https:// www.paho.org/bra/index.php?option=com_conte nt\&view=article\&id=6127: opas-oms-lanca-versaoem-portugues-de-guia-com-recomendacoes-deavaliacao-e-manejo-clinico-de-condicoes-mentaisneurologicas-e-uso-de-substancias\&Itemid=839

2. Gilbert M, Pullano G, Pinotti F, Valdano E, Polleto C, Boelle PY, et al. Preparedness and vulnerability of African countries against importations of COVID19: a modelling study. Lancet. 2020;395:871-7. DOI: https://doi.org/10.1016/S0140-6736(20)30411-6

3. Wu F, Zhao S, Yu B, Chen YM, Wang W, Song ZG, et al. A new coronavirus associated with human respiratory disease in China. Nature. 2020;579(7798):265-9. DOI: https://doi. org/10.1038/s41586-020-2008-3
4. Couto PLC, Gomes AMT, Erdmann AL, Brito OO, Nogueira VPF, Porcino C, et al. Correlação entre marcadores de vulnerabilidade social frente ao uso do preservativo por trabalhadoras sexuais. Saúde e Pesqui. 2019;12(3):591-9. DOI: https://doi. org/10.17765/2176-9206.2019v12n3p591-599

5. Ayres JRCM, França Júnior I, Calazans GJ, Saletti Filho HC. O Conceito de Vulnerabilidade e as Práticas de Saúde: novas perspectivas e desafios. In: Czeresnia D, Freitas CM, organizadores. Tratado de Saúde Coletiva. São Paulo: Hucitec; 2012. p. 117-39.

6. Couto PLS, Montalvão BPC, Vieira ARS, Vilela ABA, Marques SC, Gomes AMT, et al. Social representations of female sex workers about their sexuality. Invest Educ Enfer. 2020;38(1):e03. DOI: https://doi.org/10.17533/udea.iee.v38n1e03

7. Thng C, Blackledge E, Mclver R, Watchirs Smith L, McNulty A. Private sex workers' engagement with sexual health services: an online survey. Sexual Health. 2018;15(1):93-5. DOI: https://doi. org/10.1071/SH16243

8. Leite GS, Murray L, Lenz F. O Par e o Ímpar: o potencial de gestão de risco para a prevenção de DST/HIV/AIDS em contextos de prostituição. Rev bras epidemiol. 2015;18(Suppl 1):7-25. DOI: https://doi.org/10.1590/1809-4503201500050003

9. Cia CD. Pensar el Trabajo Social en el contexto del COVID-19. Hamartia [Internet]. 2020 abr 9. [cited 2020 Mar 27]. Available from: http://www.hamartia. com.ar/2020/04/10/trabajo-social-covid19/

10. Red de Mujeres Trabajadoras Sexuales de Latino America y el Caribe. Llamado de la cidh a los estados para que nos incluyan a las mujeres trabajadoras sexuales dentro del enfoque de sus respuestas ante la crisis por la pandemia. Bogotá (COL): Redtrasex; 2020 [cited 2020 Mar 27]. Available from: https://www.redtrasex.org/ Llamado-de-la-CIDH-a-los-Estados

11. Gomes AMT. A vulnerabilidade como elemento organizador do cuidado de enfermagem no contexto do HIV/Aids: conceitos, processos e representações sociais [tese]. Rio de Janeiro (RJ): Universidade do Estado do Rio de Janeiro; 2011.

12. Waldow VR, Borges RF. O processo de cuidar sob a perspectiva da vulnerabilidade. Rev Latino-Am Enfermagem. 2008;16(4):765-71. DOI: https://doi. org/10.1590/S0104-11692008000400018

13. Torralba I, Roselló F. Antropologia do cuidar. Petrópolis: Vozes; 2009. 
14. Oviedo RAM, Czeresnia D. O conceito de vulnerabilidade e seu caráter biossocial. Interface (Botucatu). 2015;19(53):237-50. DOI: https://doi. org/10.1590/1807-57622014.0436

15. Schumacher AA, Puttini RF, Nojimoto T. Vulnerabilidade, reconhecimento e saúde da pessoa idosa: autonomia intersubjetiva e justiça social. Saúde debate [Internet]. 2013 [cited 2020 Mar 27];37(97):281-93. Available from: https:// www.scielo.br/pdf/sdeb/v37n97/v37n97a10.pdf

16. Pires RRC. Os efeitos sobre grupos sociais e territórios vulnerabilizados das medidas de enfrentamento à crise sanitária da Covid-19: propostas para o aperfeiçoamento da ação pública. Brasília: Instituto de Pesquisa Econômica Aplicada; 2020 [cited 2020 Mar 20]. Available from: http:// repositorio.ipea.gov.br/handle/11058/9839

17. Sevalho G. O conceito de vulnerabilidade e a educação em saúde fundamentada em Paulo Freire. Interface (Botucatu). 2018;22(64):177-88. DOI: https://doi.org/10.1590/1807-57622016.0822

18. Hirata H. Gênero, classe e raça: interserccionalidade e consubstancialidade das relações sociais. Tempo social. 2014;26(1):61-73. DOI: https://doi. org/10.1590/S0103-20702014000100005

19. Kowarick L. Viver em risco-sobre a vulnerabilidade socioeconômica e civil. São Paulo: Editora 34; 2009.

20. Shannon K, Goldenberg SM, Deering K, Strathdee SA. HIV infection among female sex workers in concentrated and high prevalence epidemics: why a structural determinants framework is needed. Curr opin HIV AIDS. 2014;9(2):174-82. DOI: https://doi.org/10.1097/COH.0000000000000042

21. Porto MFS, Rocha DF, Finamore R. Saúde coletiva, território e conflitos ambientais: bases para um enfoque socioambiental crítico. Ciênc saúde coletiva. 2014;19(10):4071-80. DOI: https://doi. org/10.1590/1413-812320141910.09062014

22. Bekker LG, Johnson L, Cowan F, Overs C, Besada D, Hillier S, et al. Combination HIV prevention for female sex workers: what is the evidence?. Lancet. 2015;385(9962):72-87. DOI: https://doi.org/10.1016/S0140-6736(14)60974-0

23. Sabroza PC. Estudos epidemiológicos na perspectiva do aumento da vulnerabilidade dos sistemas sócio-ambientais brasileiros. Epidemiol Serv Saúde 2007;16(4):229-33. DOI: http://dx.doi. org/10.5123/S1679-49742007000400001

24. Beauvoir S. O Segundo Sexo: a experiência vivida. Rio de Janeiro (RJ): Nova Fronteira; 2016.

25. Lobo BNL, Galvão L, Souza SA. Impactos na vida das trabalhadoras sexuais de Belo Horizonte: territorialidade, precariedade e reconhecimento. In: Dadalto L, coordenadora. Bioética e COVID19. Indaiatuba: Foco; 2020. p. 270-91.

26. Piscitelli A. Corporalidade em Confronto: Brasileiras na indústria do sexo na Espanha. Rev bras ciênc soc. 2007;22(64):17-32. DOI: https://doi. org/10.1590/S0102-69092007000200002

27. Pasini E. Limites simbólicos corporais na prostituição feminina. Cad Pagu [Internet]. 2015 [cited 2020 Mar 27];14:181-200. Available from: https://periodicos.sbu.unicamp.br/ojs/index.php/ cadpagu/article/view/8635351

28. Broqua C, Deschamps C, Kraus C. Transactions sexuelles et imbrication des rapports de pouvoir. In: Broqua C, Deschamps C, Kraus C. L'échange économico-sexuel. Paris: Éditions EHESS; 2014. p. 45-66.

29. Deschamps C. Le sexe et l'argent des trottoirs. Paris: Hachette Littératures; 2006.

30. Organização das Nações Unidas. Profissionais do sexo não devem ser deixadas para trás na resposta à COVID-19, alerta UNAIDS. Brasília (DF); 2020 [cited 2020 Mar 27]. Available from: https:// nacoesunidas.org/profissionais-do-sexo-naodevem-ser-deixadas-para-tras-na-resposta-a-covid19-alerta-unaids/

Recebido: 16 de Junho de 2020

Aprovado: 7 de Julho de 2020

Publicado: 26 de novembro de 2020

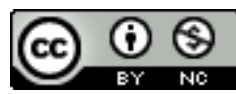

A Revista Baiana de Enfermagem utiliza a Licença Creative Commons - Atribuição-NãoComercial 4.0 Internacional. https://creativecommons.org/licenses/by-nc/4.0/

Este artigo é de acesso aberto distribuído sob os termos da Licença Creative Commons (CC BY-NC). Esta licença permite que outros remixem, adaptem e criem a partir do seu trabalho para fins não comerciais. Embora os novos trabalhos tenham de lhe atribuir o devido crédito e não possam ser usados para fins comerciais, os usuários não têm de licenciar esses trabalhos derivados sob os mesmos termos. 